\title{
Microscopic-macroscopic approach for binding energies with the Wigner-Kirkwood method. II. Deformed nuclei
}

\author{
A. Bhagwat, ${ }^{1}$ X. Viñas, ${ }^{2}$ M. Centelles, ${ }^{2}$ P. Schuck, ${ }^{3,4}$ and R. Wyss ${ }^{5}$ \\ ${ }^{1}$ UM-DAE Centre for Excellence in Basic Sciences, Mumbai 400 098, India \\ ${ }^{2}$ Departament d'Estructura i Constituents de la Matèria and Institut de Ciències del Cosmos, Facultat de Física, \\ Universitat de Barcelona, Diagonal 645, E-08028 Barcelona, Spain \\ ${ }^{3}$ Institut de Physique Nucléaire, IN2P3-CNRS, Université Paris-Sud, F-91406 Orsay-Cédex, France \\ ${ }^{4}$ Laboratoire de Physique et Modélisation des Milieux Condensés, CNRS and Université Joseph Fourier, 25 Avenue des Martyrs, \\ Boîte Postale 166, F-38042 Grenoble Cedex 9, France \\ ${ }^{5}$ KTH (Royal Institute of Technology), Alba Nova University Center, Department of Nuclear Physics, S-10691 Stockholm, Sweden
}

(Received 29 June 2012; published 12 October 2012)

\begin{abstract}
The binding energies of deformed even-even nuclei have been analyzed within the framework of a recently proposed microscopic-macroscopic model. We have used the semiclassical Wigner-Kirkwood $\hbar$ expansion up to fourth order, instead of the usual Strutinsky averaging scheme, to compute the shell corrections in a deformed Woods-Saxon potential including the spin-orbit contribution. For a large set of 561 even-even nuclei with $Z \geqslant 8$ and $N \geqslant 8$, we find an rms deviation from the experiment of $610 \mathrm{keV}$ in binding energies, comparable to the one found for the same set of nuclei using the finite range droplet model of Möller and Nix (656 keV). As applications of our model, we explore its predictive power near the proton and neutron drip lines as well as in the superheavy mass region. Next, we systematically explore the fourth-order Wigner-Kirkwood corrections to the smooth part of the energy. It is found that the ratio of the fourth-order to the second-order corrections behaves in a very regular manner as a function of the asymmetry parameter $I=(N-Z) / A$. This allows us to absorb the fourth-order corrections into the second-order contributions to the binding energy, which enables us to simplify and speed up the calculation of deformed nuclei.
\end{abstract}

DOI: 10.1103/PhysRevC.86.044316

PACS number(s): 21.10.Dr, 21.60.-n

\section{INTRODUCTION}

The models of nuclear masses are continuously challenged by advances in experimental techniques, which nowadays are extending the nuclear chart to previously unexplored regions of exotic isotopes and superheavy elements. The theoretical description of nuclear masses takes place primarily along two main approaches. On the one hand, in the microscopic nuclear models, the nuclear binding energy is obtained from calculations with energy density functionals based on effective nuclear interactions [1-3]. In the microscopic-macroscopic (mic-mac) models [2,4,5], the nuclear binding energy is obtained as the sum of a part that varies smoothly with the number of nucleons plus an oscillatory correction originated by the quantum effects. The smooth part of the mic-mac models is obtained from a liquid-drop model approach, whereas the shell correction is usually evaluated by the Strutinsky averaging method in an external potential well.

In our previous works [6,7], we have demonstrated that the Strutinsky average can be replaced by the semiclassical energy computed by means of the Wigner-Kirkwood (WK) $\hbar$ expansion of the one-body partition function [8-15], in order to evaluate the shell corrections of a system of $N$ neutrons and $Z$ protons at zero temperature in an external potential. There are some reasons supporting this choice as we have discussed in Ref. [6]. On the one hand, it has been shown that the Strutinsky level density is an approximation to the WK level density [16]. On the other hand, the WK $\hbar$ expansion of the density matrix has a variational content and it is possible to establish a variational theory based on a strict $\hbar$ expansion $[15,17]$. We shall point out that the WK expansion is also well suited to deal with nuclei close the drip lines. Although the WK level density exhibits a well known $\varepsilon^{-1 / 2}$ divergence as $\varepsilon \rightarrow 0$ for a potential that vanishes at large distances, integrated moments of the level density, such as the energy and the accumulated level density, are well behaved in the $\varepsilon \rightarrow 0$ limit, as has been demonstrated in Ref. [15]. It has been shown that these shell corrections, along with a simple six-parameter liquid-drop formula, yield a good description of ground-state masses of spherical nuclei spanning the entire periodic table [6]. The model has also been applied to calculate the binding energies of few deformed nuclei, with a good degree of success [7]. In the present work, we extend the work reported earlier [6] to the deformed nuclei and explore the predictions of the model in exotic scenarios such as drip-line nuclei and the superheavy region. In this work, we mainly restrict our attention to the even-even nuclei.

One of the important conclusions of Ref. [6] is that in this model it is necessary to carry out the WK expansion up to the fourth order in $\hbar$ to obtain accurate shell corrections, which implies that in this case one needs to work out derivatives of the single-particle potentials (nuclear potential, Coulomb potential, as well as the spin-orbit potential) up to the fourth order, which is a rather cumbersome task. Therefore, this gives rise to an interesting and important question: can the effects of the fourth-order corrections to the binding energy be absorbed into the second-order ones? This question is important from a theoretical as well as a practical point of view. Theoretically, this would imply that the WK series has been partially resummed, whereas from a practical point of view, it implies that it is sufficient to expand the one-body partition 
function up to second order in $\hbar$ to obtain shell corrections with comparable accuracy.

The absorption, if possible, would imply that there is a factor (we denote the factor by $\alpha$ ), which may be a function of mass number, charge number, neutron number, or combinations thereof, defined as

$$
\alpha=1+\frac{E\left(\hbar^{4}\right)}{E\left(\hbar^{2}\right)}
$$

such that

$$
E\left(\hbar^{2}\right)+E\left(\hbar^{4}\right)=\alpha E\left(\hbar^{2}\right),
$$

where $E\left(\hbar^{2}\right)$ and $E\left(\hbar^{4}\right)$, respectively, are second- and fourthorder WK corrections to energy. This is an important issue discussed in the present article.

We summarize the essential details of the semiclassical Wigner-Kirkwood expansion of the one-body partition function in Sec. II. The detailed results and their analysis forms the subject matter of Sec. III. The parameters of the macroscopic part of our mic-mac model, which also includes curvature correction [5] and the Wigner term [5], have been obtained by minimizing the $\chi^{2}$ value of the energies using a selected set of 561 even-even deformed and spherical nuclei. The ability of this mic-mac model to describe nuclei in the exotic scenarios is explored in Sec. IV. On the one hand, masses of very proton-rich nuclei, measured recently [18], are compared with the predictions of our model. On the other hand, the upper limit of the outer crust in neutron stars is studied, which involves nuclei near the neutron drip line. Finally, we explore the superheavy region, and compare the theoretical alpha-decay $Q$ values and the corresponding half-lives with the experimental values [19]. The systematic investigation of the absorption factor $\alpha$ as defined above is contained in Sec. V. The summary and conclusions are given in the last section.

\section{FORMULATION}

For a system of $N$ noninteracting Fermions at zero temperature in a given external potential, the quantal one-body partition function is given by

$$
Z(\beta)=\operatorname{Tr}(\exp (-\beta \hat{H})) .
$$

The Hamiltonian of the system $(\hat{H})$ is expressed as

$$
\hat{H}=\frac{-\hbar^{2}}{2 m} \nabla^{2}+V(\vec{r})+\hat{V}_{L S}(\vec{r}),
$$

with $V(\vec{r})$ being the one-body central potential and $\hat{V}_{L S}(\vec{r})$ the spin-orbit interaction. The replacement of the Hamiltonian in the above equations by the corresponding classical Hamiltonian leads to the well known Thomas-Fermi equations for particle number and total energy. The Wigner-Kirkwood semiclassical expansion amounts to expansion of the quantal one-body partition function in powers of Planck's constant $\hbar$, yielding systematic corrections to the Thomas-Fermi energy and particle number [8-13].

As stated before, in this work, we carry out the WK expansion up to the fourth order in $\hbar$. With the spin-orbit interaction, the WK expansion of the partition function can be written schematically as

$$
Z_{W K}^{(4)}(\beta)=Z^{(4)}(\beta)+Z_{S O}^{(4)}(\beta),
$$

where $Z^{(4)}(\beta)\left(Z_{S O}^{(4)}(\beta)\right)$ is the WK partition function for the central potential (spin-orbit part). The explicit expressions for these partition functions can be found in Refs. [6,10].

The level density $g_{W K}$, the particle number $N$, and the energy $E_{W K}$ are obtained by appropriate Laplace inversion of the WK partition function, as follows:

$$
\begin{aligned}
g_{W K}(\epsilon) & =\mathcal{L}_{\epsilon}^{-1} Z_{W K}^{(4)}(\beta), \\
N & =\mathcal{L}_{\lambda}^{-1}\left(\frac{Z_{W K}^{(4)}(\beta)}{\beta}\right),
\end{aligned}
$$

and

$$
E_{W K}=\lambda N-\mathcal{L}_{\lambda}^{-1}\left(\frac{Z_{W K}^{(4)}(\beta)}{\beta^{2}}\right),
$$

Here, $\lambda$ is the chemical potential, determined to ensure the correct particle number.

The focus of the present article being the WK energy, we present the explicit expressions for the WK energies alone. Following Jennings et al. [10], the energy [Eq. (8)] can be written as

$$
E_{W K}=\lambda N-\left(E_{\hbar^{0}}^{C N}+E_{\hbar^{2}}^{C N}+E_{\hbar^{4}}^{C N}\right)-\left(E_{\hbar^{2}}^{S O}+E_{\hbar^{4}}^{S O}\right),
$$
where $E_{\hbar^{k}}^{C N}$ denote the contribution to the energy of order $\hbar^{k}$ arising from Laplace inversion $\mathcal{L}_{\lambda}^{-1}\left(Z^{(4)}(\beta) / \beta^{2}\right)$. On the other hand, $E_{\hbar^{k}}^{S O}$ are corrections to the energy of order $\hbar^{k}$ due to Laplace inversion $\mathcal{L}_{\lambda}^{-1}\left(Z_{S O}^{(4)}(\beta) / \beta^{2}\right)$. The explicit expression are as follows (see Ref. [6] for further details):

$$
\begin{aligned}
& E_{\hbar^{0}}^{C N}=\frac{1}{3 \pi^{2}}\left(\frac{2 m}{\hbar^{2}}\right)^{3 / 2} \int d \vec{r}\left\{\frac{2}{5}(\lambda-V)^{5 / 2}\right\} \Theta(\lambda-V), \\
& E_{\hbar^{2}}^{C N}=-\frac{1}{24 \pi^{2}}\left(\frac{2 m}{\hbar^{2}}\right)^{1 / 2} \int d \vec{r}\left\{(\lambda-V)^{1 / 2} \nabla^{2} V\right\} \Theta(\lambda-V), \\
& E_{\hbar^{4}}^{C N}=-\frac{1}{5760 \pi^{2}}\left(\frac{\hbar^{2}}{2 m}\right)^{1 / 2}\left[\int d \vec{r}(\lambda-V)^{-1 / 2}\left\{7 \nabla^{4} V\right\}+\frac{1}{2} \int d \vec{r}(\lambda-V)^{-3 / 2}\left\{5\left(\nabla^{2} V\right)^{2}+\nabla^{2}(\nabla V)^{2}\right\}\right] \Theta(\lambda-V), \\
& E_{\hbar^{2}}^{S O}=\frac{\kappa^{2}}{6 \pi^{2}}\left(\frac{2 m}{\hbar^{2}}\right)^{1 / 2} \int d \vec{r}\left\{(\lambda-V)^{3 / 2}(\nabla f)^{2}\right\} \Theta(\lambda-V),
\end{aligned}
$$




$$
\begin{aligned}
E_{\hbar^{4}}^{S O}= & \frac{1}{48 \pi^{2}}\left(\frac{\hbar^{2}}{2 m}\right)^{1 / 2} \int d \vec{r}(\lambda-V)^{1 / 2}\left[\kappa^{2}\left\{\frac{1}{2} \nabla^{2}(\nabla f)^{2}-\left(\nabla^{2} f\right)^{2}+\nabla f \cdot \nabla\left(\nabla^{2} f\right)-\frac{(\nabla f)^{2} \nabla^{2} V}{2(\lambda-V)}\right\}\right. \\
& \left.-2 \kappa^{3}\left\{(\nabla f)^{2} \nabla^{2} f-\frac{1}{2} \nabla f \cdot \nabla(\nabla f)^{2}\right\}+2 \kappa^{4}(\nabla f)^{4}\right] \Theta(\lambda-V) .
\end{aligned}
$$

In these expressions, $V$ is the mean field, $f$ is the spin-orbit form factor, $\kappa$ is the strength of the spin-orbit interaction, and $\lambda$ is the chemical potential.

The shell corrections, which are the difference between the quantum mechanical and the corresponding averaged energies, can now be obtained by subtracting $E_{W K}$ from the quantum mechanical energy. For our calculations we choose a WoodsSaxon potential as mean field and a suitable Woods-Saxon form factor in the spin-orbit sector. These potentials are generalized for taking into account deformation effects, and their corresponding parameters are given in Ref. [6]. The Coulomb potential has been obtained by folding the proton density distribution with the Coulomb interaction [6]. In the microscopic part we have also included pairing correlations using the Lipkin-Nogami scheme [20-22], as described in detail in Ref. [6].

\section{CALCULATION OF BINDING ENERGIES}

In the present work, we generalize the liquid-drop formula employed in Ref. [6] by adding a deformation-dependent curvature energy term and the Wigner term. The curvature energy term is found to be important in improving the agreement achieved between calculations and the corresponding experimental binding energies [5]. The Wigner term is expected to be important for light nuclei as well as to describe nuclei close to the proton drip line. Therefore, the modified liquid-drop formula used in this work reads

$$
\begin{aligned}
E_{\mathrm{LDM}}= & a_{v}\left[1+\frac{4 k_{v}}{A^{2}} T_{z}\left(T_{z}+1\right)\right] A \\
& +a_{s}\left[1+\frac{4 k_{s}}{A^{2}} T_{z}\left(T_{z}+1\right)\right] A^{2 / 3} \\
& +a_{\mathrm{cur}}\left[1+\frac{4 k_{\mathrm{cur}}}{A^{2}} T_{z}\left(T_{z}+1\right)\right] A^{1 / 3}+\frac{3 Z^{2} e^{2}}{5 r_{0} A^{1 / 3}} \\
& +\frac{C_{4} Z^{2}}{A}+E_{W},
\end{aligned}
$$

where the terms respectively represent volume energy, surface energy, curvature energy, Coulomb energy, correction to Coulomb energy due to surface diffuseness of charge distribution, and the Wigner energy. The coefficients $a_{v}, a_{s}$, $a_{\text {cur }}, k_{v}, k_{s}, k_{\text {cur }}, r_{0}$, and $C_{4}$ are free parameters; $T_{z}$ is the third component of isospin, and $e$ is the electronic charge.

Several parametrizations of the Wigner term are available in the literature (see, for example, Refs. [2,5,23]). Here, we adopt the following ansatz for the Wigner term with a cutoff on charge and mass numbers:

$$
E_{W}=w_{1} \exp \left\{-w_{2}\left|\frac{N-Z}{A}\right|\right\} \Theta(Z-20) \Theta(A-40),
$$

where $w_{1}$ and $w_{2}$ are free parameters. The cutoffs on charge and mass numbers have been introduced since it is expected that the Wigner term will make significant contributions for nuclei with low masses.

The Coulomb, surface, and curvature terms appearing in the liquid-drop formula, as defined above in Eq. (15), need to be modified for the deformed shapes. In particular, the Coulomb term is multiplied by

$$
\begin{aligned}
\mathcal{F}_{c} & =\frac{15}{32 \pi^{2}} \int \frac{1}{\left|\vec{r}-\vec{r}^{\prime}\right|} d \vec{r} d \vec{r}^{\prime} \\
& =\frac{-15}{64 \pi^{2}} \int\left|\vec{r}-\vec{r}^{\prime}\right| d \vec{S} \cdot d \vec{S}^{\prime},
\end{aligned}
$$

where the symbols have their usual meanings. Notice that the integrals have been carried out over nuclear volume, and the lengths have been measured in units of the radius parameter $R_{o}$ of the nucleus with zero deformation. The transformation from six-dimensional to four-dimensional integrals has been accomplished by following the technique developed by Kurmanov et al. [24]. The surface term, on the other hand, is simply modified by the ratio of the deformed to the corresponding spherical surface areas. The curvature energy term, too, needs to be modified to take the deformation effects into account. The modified curvature energy $\left(E_{\text {cur }}\right)$ reads

$$
E_{\text {cur }}=\frac{E_{\text {cur }}^{0}}{8 \pi} \int_{\Omega}\left(\frac{1}{R_{1}}+\frac{1}{R_{2}}\right) d S,
$$

where $E_{\text {cur }}^{0}$ is curvature energy at zero deformation; $R_{1}$ and $R_{2}$ are the principal radii of curvature of the nuclear surface (in the units of $R_{o}$ ), defined by $r=r_{s}$; and $d S$ refers to the area element of the nuclear surface. The surface parametrization assumed in the present work is given by

$$
r_{s}=C R_{0}\left(1+\sum_{\lambda, \mu} \alpha_{\lambda, \mu} Y_{\lambda, \mu}\right)
$$

Here, the $Y_{\lambda, \mu}$ functions are the usual spherical harmonics and the constant $C$ is the volume conservation factor (the volume enclosed by the deformed surface should be equal to the volume enclosed by an equivalent spherical surface of radius $R_{0}$ ):

$$
C=\left[\frac{1}{4 \pi} \int_{\Omega}\left\{1+\sum_{\lambda, \mu} \alpha_{\lambda, \mu} Y_{\lambda, \mu}(\Omega)\right\}^{3} d \Omega\right]^{-1 / 3} .
$$

The term $Z^{2} / A$, which is the correction to Coulomb energy due to surface diffuseness of the charge distribution, does not have any explicit deformation dependence. This is because the distance function chosen here is such that the surface thickness is the same in all directions (see the discussion about this in Ref. [6]). 
The total binding energy of a nucleus with $N$ neutrons, $Z$ protons, and deformation parameters $\beta_{2}, \beta_{4}$, and $\gamma$ is given by

$$
\begin{aligned}
E\left(N, Z, \beta_{2}, \beta_{4}, \gamma\right)= & E_{\mathrm{LDM}}\left(N, Z, \beta_{2}, \beta_{4}, \gamma\right) \\
& +\eta \delta E\left(N, Z, \beta_{2}, \beta_{4}, \gamma\right),
\end{aligned}
$$

where $\delta E$ represents the microscopic part of the binding energy (shell correction plus pairing energy). The microscopic part has been multiplied by a factor $\eta$, which is chosen to be 0.85 . One of the reasons for introducing such a factor is that the Coulomb potential used in the present work is less repulsive near $r=0$ than the corresponding value obtained by using the hard sphere approximation, used in the fit of proton mean field (see discussion on this point in Ref. [6]).

The free parameters of the liquid-drop formula are determined by minimizing the $\chi^{2}$ value in comparison with the experimental binding energies [25]:

$$
\chi^{2}=\frac{1}{n} \sum_{j=1}^{n}\left[\frac{E\left(N_{j}, Z_{j}\right)-E_{\mathrm{expt}}^{(j)}}{\Delta E_{\mathrm{expt}}^{(j)}}\right]^{2},
$$

where $E\left(N_{j}, Z_{j}\right)$ is the calculated total binding energy for the given nucleus, $E_{\text {expt }}^{(j)}$ is the corresponding experimental value [25], and $\Delta E_{\text {expt }}^{(j)}$ is the uncertainty in $E_{\text {expt }}^{(j)}$. In the present fit, for simplicity, $\Delta E_{\text {expt }}^{(j)}$ is set to $1 \mathrm{MeV}$.

To obtain these parameters we proceed as follows. We start by setting in the liquid-drop mass formula (15) the values obtained in our spherical calculation [6]. Explicitly, these values are $a_{v}=-15.841 \mathrm{MeV}, a_{s}=19.173 \mathrm{MeV}, k_{v}=$ $-1.951, k_{S}=-2.577, r_{0}=1.187 \mathrm{fm}$, and $C_{4}=1.247 \mathrm{MeV}$. Next, we choose a set of 561 even-even nuclei with $Z \geqslant 8$ and $N \geqslant 8$, the list of which may be found in Ref. [26]. This set comprises doubly magic, semimagic, as well as open-shell nuclei, many of which are expected to be deformed. The main task now is to determine the liquid-drop parameters as well as the optimal deformation parameters. The calculation proceeds in the following steps:

(i) Assuming the previously reported [6] values of the liquid-drop parameters, the binding energies of these nuclei are obtained by minimizing on a range of $\beta_{2}$ values ( $\beta_{4}$ is set to zero in this step). This gives a preliminary estimation of $\beta_{2}$. Next, keeping this $\beta_{2}$ fixed, $\beta_{4}$ is varied to obtain minimum energy. Thus, we now have preliminary values of both the deformation parameters.

(ii) In the next step, keeping the deformation parameters fixed as obtained in the earlier step, the liquid-drop parameters are fitted by minimizing $\chi^{2}$.

(iii) With the new values of liquid-drop parameters, the deformation parameters are obtained once again as described in step (i), followed by a final refit to the liquid-drop parameters.

The numerical values of the new constants of the liquiddrop formula obtained through this minimization procedure are $a_{v}=-15.435 \mathrm{MeV}, a_{s}=16.673 \mathrm{MeV}, a_{\text {cur }}=3.161 \mathrm{MeV}$, $k_{v}=-1.874, k_{S}=-2.430, k_{\mathrm{cur}}=0$ (see discussion below), $r_{0}=1.219 \mathrm{fm}, C_{4}=0.963 \mathrm{MeV}, w_{1}=-2.762 \mathrm{MeV}$, and $w_{2}=3.725$. The values of volume, surface, and Coulomb coefficients differ from those reported earlier [6], primarily due to the inclusion of curvature and Wigner terms and the deformation effects. The curvature term, as described earlier, depends on the mean curvature of the nucleus, which is a function of the geometry of the nuclear surface. Therefore, the curvature energy, a priori, is expected to modify the surface energy term as well as the $Z^{2} / A$ term, which is the correction due to the surface diffuseness of the charge density term. The somewhat smaller value of the volume coefficient reported here is not surprising. The reduction is due to the influence of the curvature term, as has been found also by Pomorski and Dudek (see Table I of Ref. [5]).

It is to be noted that the coefficient of the isospin-dependent term in the curvature energy is very difficult to determine with experimental masses. In our case the resulting statistical error in the corresponding parameter turns out to be more than $50 \%$ of the numerical value of the coefficient. Further, this term is found to weaken the strength of the isospin-dependent term in the surface energy by a factor of 5 . The isospin dependence in the curvature term, therefore, has been dropped from the present investigation.

The rms deviation of the calculated binding energies with respect to those obtained by experiment is $610 \mathrm{keV}$. The Möller-Nix calculations [27], for the same set of nuclei, yield a deviation of $656 \mathrm{keV}$. The explicit values of binding energies of our selected set of 561 even-even nuclei used in the minimization procedure can be found in Ref. [26]. The present calculation establishes that our model is indeed capable of reproducing binding energies of deformed nuclei as well, with excellent accuracy. The difference between the calculated and the corresponding evaluated [25] binding energies is presented in Fig. 1. The corresponding differences obtained for the Möller-Nix calculations are presented in the same figure for comparison. The excellent agreement found between the calculations and experiment is amply clear from the figure.

We next present and discuss the results obtained for $\mathrm{Sr}, \mathrm{Sn}$, $\mathrm{Gd}$, and Po isotopes as illustrative examples. The differences

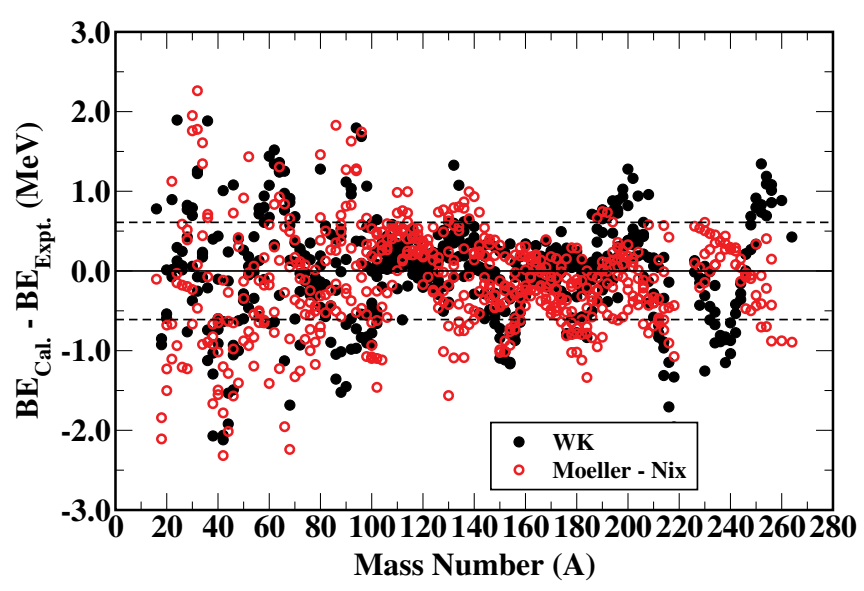

FIG. 1. (Color online) Difference between the calculated (fitted) and the corresponding experimental [25] binding energies, as a function of mass number. The dashed horizontal lines correspond to $\delta B E=610 \mathrm{keV}$. The corresponding differences obtained by using the Möller-Nix binding energies are also presented for comparison. 

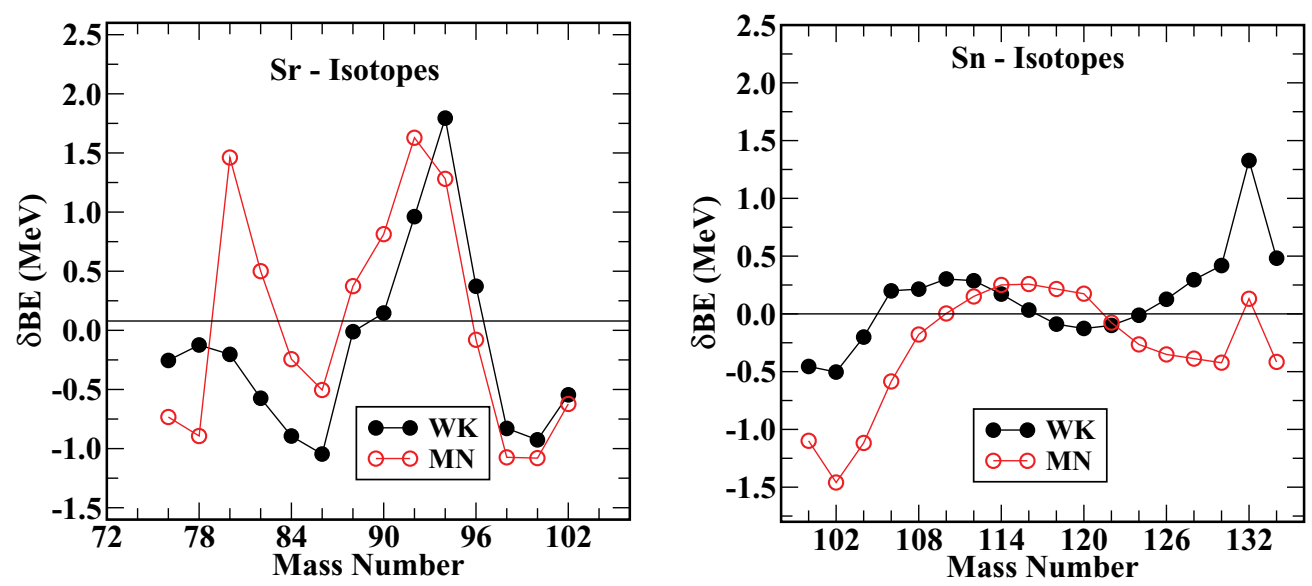

FIG. 2. (Color online) The difference between the calculated and the experimental [25] binding energies for $\mathrm{Sr}$ and $\mathrm{Sn}$ isotopes.

between the fitted and the corresponding experimental binding energies for these nuclei are plotted in Figs. 2 and 3, along with the corresponding differences obtained from the Möller-Nix calculations [27]. The figures reveal that the calculated binding energies (denoted by WK) are quite close to the experimental values. The differences are found to vary quite smoothly as a function of mass number. Next, we present the twoneutron separation energies for these chains. The two-nucleon separation energies highlight the shell structure in an isotopic chain. Correct prediction of these separation energies is crucial for determination of the drip lines. The calculated and the corresponding experimental [25] two-neutron separation energies are plotted in Figs. 4 and 5. The figures reveal that the present calculations reproduce the experimental separation energies very well and that the shell gaps are also reproduced nicely.

In addition, the systematics of deformation parameters obtained in these calculations turns out to be reasonable. As an illustrative example, we focus on the $\mathrm{Sr}-\mathrm{Zr}$ region. It is well known from the systematics of experimentally measured charge radii [28] that the charge radii increase dramatically by $2 \%$ for ${ }^{97} \mathrm{Rb},{ }^{98} \mathrm{Sr}$, and ${ }^{100} \mathrm{Zr}$, in comparison to their respective lighter isotopes. This jump may be attributed to the possibility of onset of highly deformed shapes in the ground state, around this neutron number (see, for example, Ref. [29]). Our calculations, too, reveal the existence of highly deformed ground states (with $\beta_{2} \sim 0.3$ ) around neutron number 60 , in the $\mathrm{Sr}-\mathrm{Zr}$ region. The values of $\beta_{2}$ obtained in this work for $\mathrm{Kr}$, $\mathrm{Sr}, \mathrm{Zr}$, and Mo chains are plotted in Fig. 6. The sudden change in the ground-state deformation around neutron number 60 is very clear from the figure.

Further, it is also well known that the ground states of ${ }^{72} \mathrm{Kr},{ }^{76} \mathrm{Sr}$, and ${ }^{80} \mathrm{Zr}$ have very large $(\sim 0.4)$ deformation. This is known to be due to population in the intruder $1 g_{9 / 2}$ state. Thus, the ground state of ${ }^{80} \mathrm{Zr}$ is a 12-particle 12-hole state, which is manifested again by an extremely large stable deformation in the ground state of ${ }^{80} \mathrm{Zr}$. This has been verified independently, for example, by the relativistic mean-field calculation [30], density-dependent Hartree Fock calculation with Skyrme interaction [31], as well as by the Hartree Fock band mixing calculation [32]. The deformation parameters reported in the Möller-Nix table [23], too, are consistent with the discussion above. It is gratifying to note that the present calculations, indeed, yield $\beta_{2}=-0.36,-0.41$, and 0.44 respectively, for ${ }^{72} \mathrm{Kr},{ }^{76} \mathrm{Sr}$, and ${ }^{80} \mathrm{Zr}$, which is in tune with the mean-field as well as the mic-mac Möller-Nix calculations cited above.
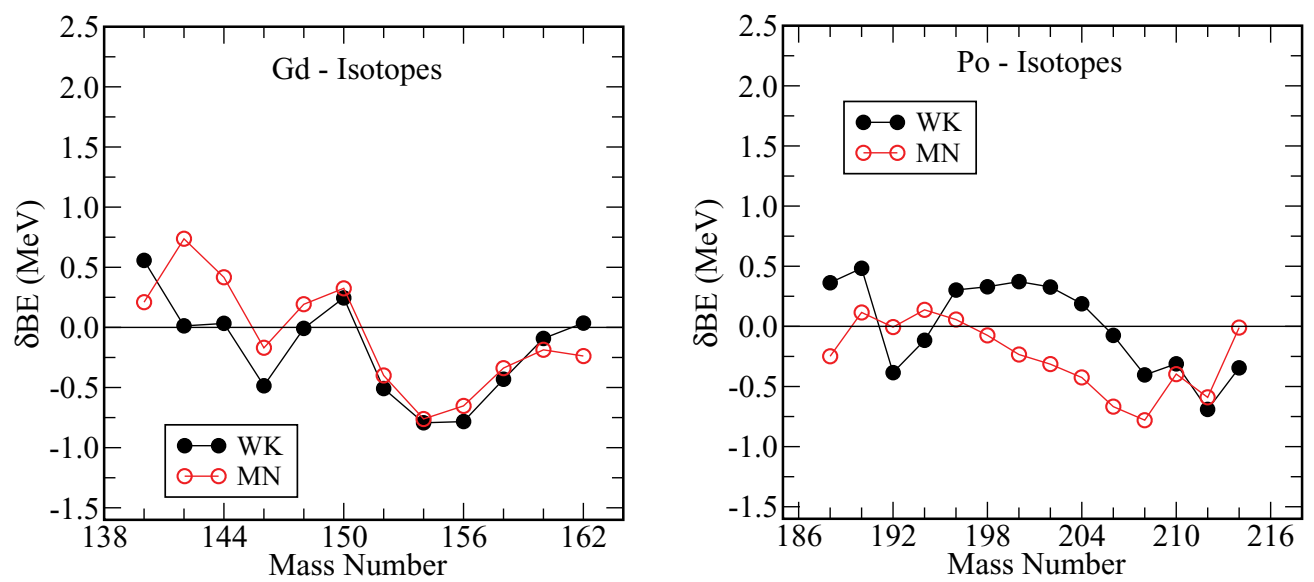

FIG. 3. (Color online) The difference between the calculated and the experimental [25] binding energies for Gd and Po isotopes. 

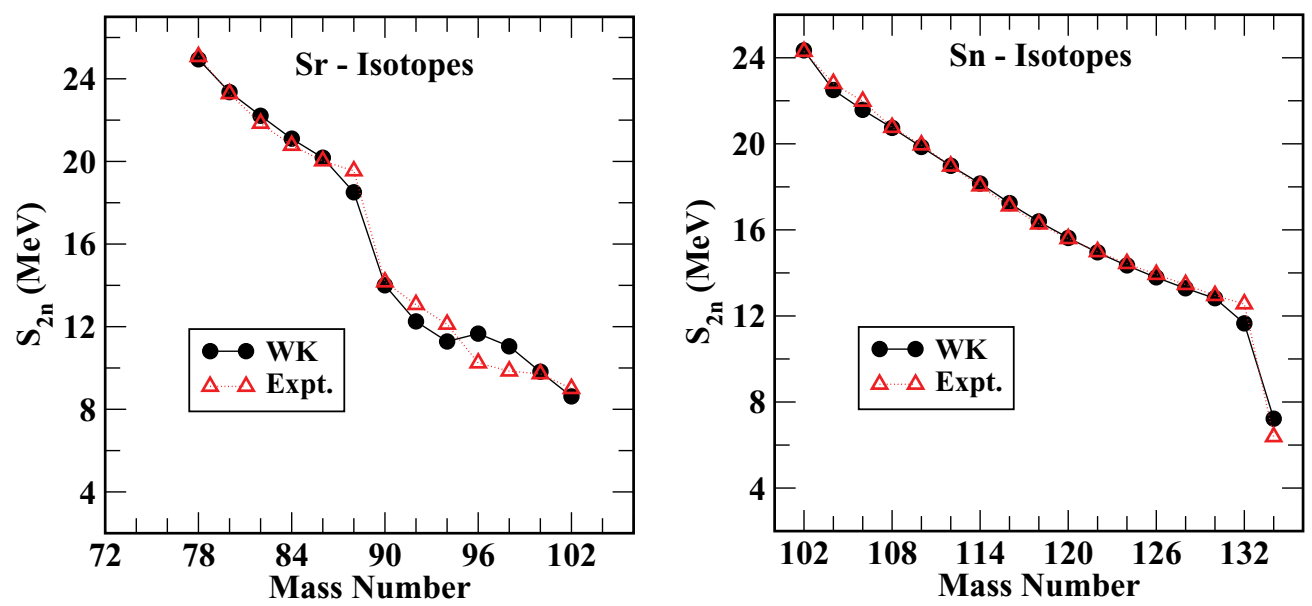

FIG. 4. (Color online) The calculated and the experimental [25] two-neutron separation energies for Sr and $\mathrm{Sn}$ isotopes.

\section{APPLICATIONS OF THE PRESENT MODEL TO NEAR-DRIP-LINE NUCLEI AND SUPERHEAVY NUCLEI}

We next test the ability of the present model to describe binding energies of the neutron-rich and neutron-deficient nuclei, as well as of the superheavy nuclei. To this end, we now present a few exploratory calculations.

\section{A. Proton drip-line nuclei in the $\mathrm{Ge}-\mathrm{Kr}$ region}

The masses of ${ }^{63} \mathrm{Ge},{ }^{65} \mathrm{As},{ }^{67} \mathrm{Se}$, and ${ }^{71} \mathrm{Kr}$ have recently been measured [18]. These nuclei are very proton rich, and are expected to be close to the drip line. Notice that these nuclei are odd-even and even-odd. In this preliminary test of our model near the proton drip line, we use the simple uniform filling approach for the calculation of the pairing energy. The calculated binding energies and one-proton separation energies $\left(S_{p}\right)$ for these nuclei, along with the corresponding experimental values [18] and those reported by Möller and Nix [27] are presented in Table I. The binding energies as well as $S_{p}$ values obtained in the present work are found to be quite close to the experiment. This indicates that the present model extrapolates reliably up to the proton drip lines. The nucleus ${ }^{65} \mathrm{As}$ is reported to be slightly unbound against proton emission with $S_{p}=-90 \pm 85 \mathrm{keV}$ [18]. Our calculation, on the other hand, yields a positive value of $S_{p}$ for ${ }^{65} \mathrm{As}$, indicating a proton bound nucleus. However, it should be noted that the separation energies are obtained by taking differences of the relevant binding energies, and hence are very sensitive to the precise details of the same. The fact that the theoretical separation energies obtained in this work differ from the corresponding experimental values only by a few hundred $\mathrm{keV}$ is quite remarkable.

\section{B. Composition of the outer crust of neutron stars}

The masses of very neutron-rich nuclei are particularly interesting for some astrophysical calculations. We next compute the composition of the outer crust of a neutron star as a further application of our present mass model. As one moves from the surface of a neutron star to its interior, the outer crust is the region comprising matter at densities between $\sim 10^{4} \mathrm{~g} / \mathrm{cm}^{3}$ and $\sim 10^{11} \mathrm{~g} / \mathrm{cm}^{3}$. Matter at those densities consists of fully ionized, neutron-rich atomic nuclei that arrange themselves in

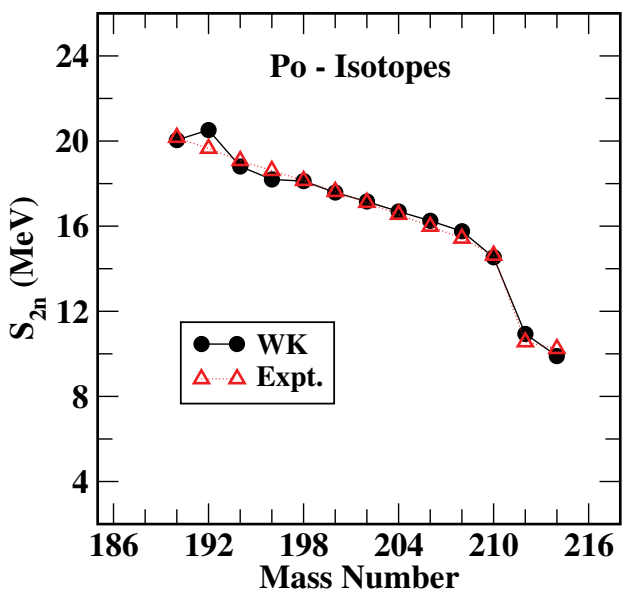

FIG. 5. (Color online) The calculated and the experimental [25] two-neutron separation energies for Gd and Po isotopes. 


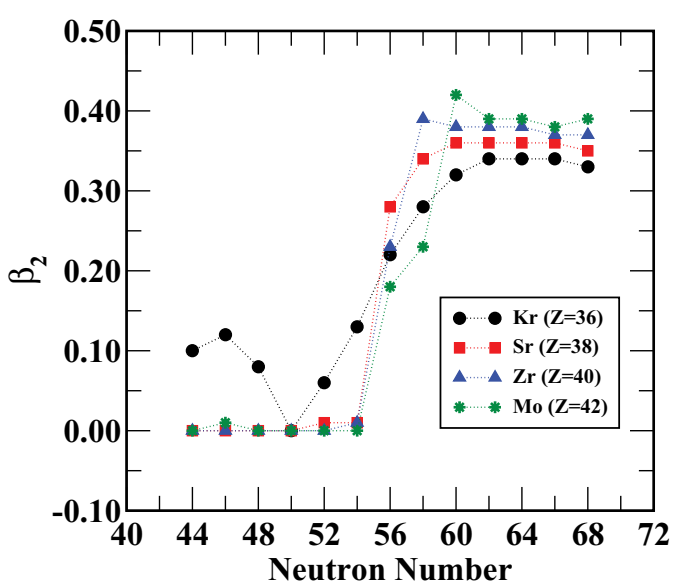

FIG. 6. (Color online) Deformation parameter $\beta_{2}$ for $\mathrm{Kr}, \mathrm{Sr}, \mathrm{Zr}$, and Mo isotopes.

the lattice sites of a Coulomb crystal embedded in a degenerate electron gas [33]. The neutron excess of the nuclei in the outer crust becomes larger with increasing matter density until neutron drip starts taking place at a density of about $4 \times 10^{11} \mathrm{~g} / \mathrm{cm}^{3}$. At that point, one leaves the outer crust and enters the so-called inner crust of the neutron star, where the atomic nuclei are immersed in an electron gas and a neutron gas.

In order to compute the composition of the outer crust we follow the usual formalism as described in Refs. [34-36] and references quoted therein. That is, we consider cold and electrically neutral matter which is assumed to be in thermodynamic equilibrium and in its absolute ground state. We calculate the Gibbs free energy of this system by adding the contributions of the nuclear, electronic, and lattice terms [34-36] and, finally, we evaluate the equilibrium composition $(Z, N)$ at a certain pressure by minimizing the obtained Gibbs free energy per nucleon.

We display our predictions for the equilibrium nuclear species present in the outer crust in Fig. 7. We perform the calculations within the range $\rho=10^{7} \mathrm{~g} / \mathrm{cm}^{3}$ to $\rho=3 \times$ $10^{11} \mathrm{~g} / \mathrm{cm}^{3}$. The variation of the neutron and proton numbers with increasing crustal density shows a structure of plateaus that are interrupted by abrupt jumps in the composition. As exemplified by the $N=50$ plateau, the prevalence of a given nucleon number over a large range of densities is related to the shell effect due to the filling of a nuclear shell. The $N=50$ neutron plateau also is very illustrative of the fact that, with

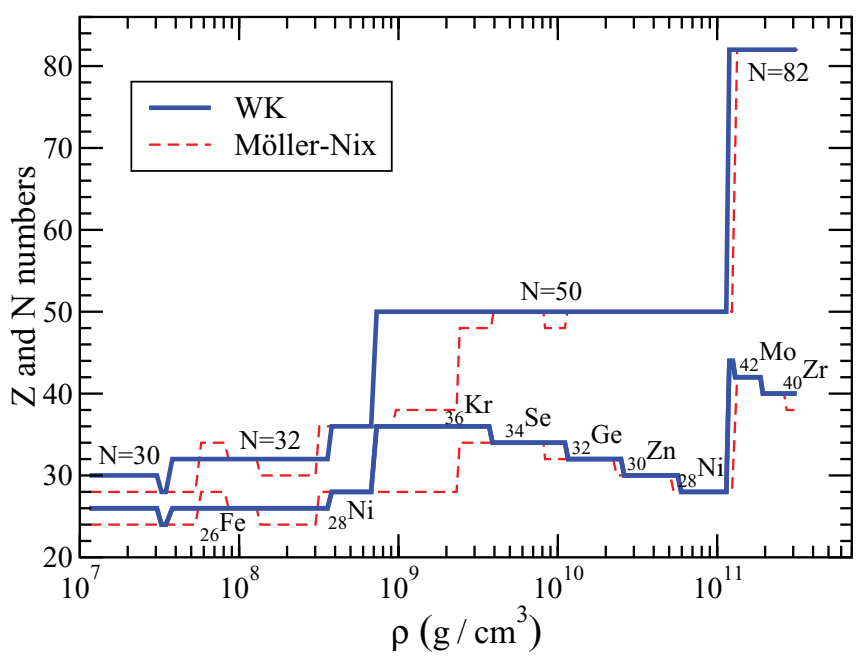

FIG. 7. (Color online) Predicted composition of the outer crust of a neutron star as a function of the density. The upper line depicts the variation of the neutron number $N$, while the lower line depicts the variation of the proton number $Z$. The composition obtained by using the Möller-Nix mass formula is also presented for comparison.

increasing density, it is energetically favorable for the nuclei of the crust to capture electrons from the degenerate electron gas. This results in increasingly neutron-rich nuclides along the neutron plateau. Eventually, the mismatch between the neutron and proton numbers is too large and the jump to the next neutron plateau takes place in an effort to reduce the penalty imposed on the system by the nuclear symmetry energy [35,36].

At low crustal densities up to about $\rho=7 \times 10^{8} \mathrm{~g} / \mathrm{cm}^{3}$, our calculations sequentially favor ${ }_{26}^{56} \mathrm{Fe},{ }_{26}^{58} \mathrm{Fe}$, and ${ }_{28}^{64} \mathrm{Ni}$ as the equilibrium nuclides (with ${ }_{24}^{52} \mathrm{Cr}$ occurring in a short density interval between ${ }_{26}^{56} \mathrm{Fe}$ and ${ }_{26}^{58} \mathrm{Fe}$ ). Once the jump to the $N=50$ plateau ensues at a density $\rho \sim 7 \times 10^{8} \mathrm{~g} / \mathrm{cm}^{3}$, our model predicts the sequence of increasingly neutron-rich isotones ${ }_{36}^{86} \mathrm{Kr},{ }_{34}^{84} \mathrm{Se},{ }_{32}^{82} \mathrm{Ge},{ }_{30}^{80} \mathrm{Zn}$, and ${ }_{28}^{78} \mathrm{Ni}$. After the ${ }_{28}^{78} \mathrm{Ni}$ nucleus, it is unfavorable to move further to ${ }_{26}^{76} \mathrm{Fe}$, and at a density $\rho \sim$ $1.2 \times 10^{11} \mathrm{~g} / \mathrm{cm}^{3}$ we find that the composition of the crust jumps to the $N=82$ plateau (where our calculations predict the occurrence of the isotones ${ }_{42}^{124} \mathrm{Mo}$ and ${ }_{40}^{122} \mathrm{Zr}$ ). We display the results obtained with the Möller-Nix mass table [23] in the same Fig. 7 for comparison. Though the overall pattern is quite similar to the results obtained with our calculated masses, the Möller-Nix mass table predicts more structure in the variation

TABLE I. The binding energies and one proton separation energies for proton rich nuclei. 'Calc.' (MN) represent the results obtained in the present work (by Möller and Nix [27]). The experimental binding energies have been obtained from mass excess values reported by Tu et al. [18]. The experimental $S_{p}$ values have been also been adopted from Ref. [18].

\begin{tabular}{|c|c|c|c|c|c|c|c|c|}
\hline & $\beta_{2}$ & $\beta_{4}$ & \multicolumn{3}{|c|}{ Binding energy $(\mathrm{MeV})$} & \multicolumn{3}{|c|}{$S_{p}(\mathrm{MeV})$} \\
\hline${ }^{63} \mathrm{Ge}$ & +0.200 & -0.010 & -529.795 & -529.266 & -530.327 & 2.557 & 3.315 & 2.210 \\
\hline${ }^{65} \mathrm{As}$ & +0.210 & -0.030 & -545.168 & -544.642 & -545.699 & 0.633 & 0.124 & -0.090 \\
\hline${ }^{67} \mathrm{Se}$ & +0.220 & -0.050 & -560.598 & -560.158 & -560.698 & 2.379 & 3.364 & 1.852 \\
\hline${ }^{71} \mathrm{Kr}$ & -0.330 & 0.010 & -592.047 & -591.219 & -591.150 & 2.304 & 3.093 & 2.184 \\
\hline
\end{tabular}


of the neutron and proton numbers with the crustal density, and the jump to the $N=50$ plateau is delayed to a little higher density. This fact suggests that in the present mass region the shell effects due to the filling of nuclear shells and subshells are somewhat weaker in the Möller-Nix mass formula than in our model.

\section{Superheavy nuclei}

Production and study of superheavy nuclei is of current interest from both theoretical [37-40] and experimental $[19,41]$ aspects. With the advent of increasingly sensitive detection methods, it is possible to identify the superheavy elements, and measure $\alpha$-decay $Q$ values precisely. The elements with $Z=118$ have been produced so far [19]. Here, we apply our mic-mac model to a few recently reported superheavy nuclei [19]. In particular, we focus on the $\alpha$-decay $Q$ values $\left(Q_{\alpha}\right)$. The binding energies of the parent as well as the daughter nuclei, necessary to obtain the $Q_{\alpha}$ values, are obtained within our mic-mac model by minimizing over the deformation $\left(\beta_{2}, \beta_{4}\right)$ mesh. The binding energy of the $\alpha$ particle is adopted from the Audi-Wapstra compilation [25]. The calculated (Calc.) as well as the experimental $Q$ values [19] are presented in Table II. We find that the calculated $Q_{\alpha}$ values are very close to the experiment. This is quite encouraging since, as in the case of the separation energies, the $Q$ values as well are obtained by taking differences between two large quantities.

The $\alpha$ decay $Q$ values can be related to the half-lives through the Viola-Seaborg relation [42]. In particular, following Oganessian [19], we adopt

$$
\log T_{1 / 2}=\frac{a Z+b}{\sqrt{Q_{\alpha}}}+c Z+d,
$$

where $Z$ is the charge number of the parent nucleus; $Q_{\alpha}$ is the $\alpha$-decay $Q$ value; and $a, b, c$, and $d$ are parameters, taken to be [19] $a=1.787, b=-21.40, c=-0.2549$, and $d=-28.42$.

TABLE II. The $\alpha$-decay $Q$ values and half-lives $\left(T_{1 / 2}\right)$ for some of the superheavy nuclei.

\begin{tabular}{|c|c|c|c|c|c|}
\hline \multirow[b]{2}{*}{$Z$} & \multirow[b]{2}{*}{$A$} & \multicolumn{2}{|c|}{$Q_{\alpha}(\mathrm{MeV})$} & \multicolumn{2}{|c|}{$T_{1 / 2}$} \\
\hline & & Calc. & Expt. & Calc. & Expt. \\
\hline 118 & 294 & 11.76 & $11.81 \pm 0.06$ & $0.56 \mathrm{~ms}$ & $0.89_{-0.31}^{+1.07} \mathrm{~ms}$ \\
\hline 116 & 293 & 10.59 & $10.69 \pm 0.06$ & $136 \mathrm{~ms}$ & $61_{-20}^{+57} \mathrm{~ms}$ \\
\hline 116 & 292 & 10.66 & $10.80 \pm 0.07$ & $89 \mathrm{~ms}$ & $18_{-6}^{+16} \mathrm{~ms}$ \\
\hline 116 & 291 & 10.89 & $10.89 \pm 0.07$ & $22 \mathrm{~ms}$ & $18_{-6}^{+22} \mathrm{~ms}$ \\
\hline 115 & 288 & 10.49 & $10.61 \pm 0.06$ & $129 \mathrm{~ms}$ & $87_{-30}^{+105} \mathrm{~ms}$ \\
\hline 115 & 287 & 11.38 & $10.74 \pm 0.09$ & $0.69 \mathrm{~ms}$ & $32_{-14}^{+155} \mathrm{~ms}$ \\
\hline 114 & 289 & 9.91 & $9.96 \pm 0.05$ & $2.7 \mathrm{~s}$ & $2.6_{-0.7}^{+1.2} \mathrm{~s}$ \\
\hline 114 & 288 & 10.26 & $10.08 \pm 0.06$ & $0.28 \mathrm{~s}$ & $0.80_{-0.16}^{+0.27} \mathrm{~s}$ \\
\hline 114 & 287 & 10.19 & $10.16 \pm 0.06$ & $0.43 \mathrm{~s}$ & $0.48_{-0.09}^{+0.16} \mathrm{~s}$ \\
\hline 113 & 283 & 10.82 & $10.26 \pm 0.09$ & $4.6 \mathrm{~ms}$ & $100_{-45}^{+490} \mathrm{~ms}$ \\
\hline 113 & 282 & 10.99 & $10.78 \pm 0.08$ & $17 \mathrm{~ms}$ & $73_{-29}^{+134} \mathrm{~ms}$ \\
\hline 111 & 280 & 9.33 & $9.87 \pm 0.06$ & $17 \mathrm{~s}$ & $3.6_{-1.3}^{+4.3} \mathrm{~s}$ \\
\hline 111 & 279 & 10.56 & $10.52 \pm 0.16$ & $5.5 \mathrm{~ms}$ & $170_{-80}^{+810} \mathrm{~ms}$ \\
\hline
\end{tabular}

The half-lives obtained by using the calculated $Q$ values are found to be in reasonable agreement with the experiment. At places, the calculations do deviate by an order of magnitude, but notice that the half-lives have very large uncertainties.

\section{SYSTEMATIC INVESTIGATION OF THE FACTOR $\alpha$}

Large-scale calculations using the proposed mic-mac model can be cumbersome and highly time consuming. Therefore, it may be very useful to look for simplifications that allow one to speed up the calculations without loss of accuracy. To this end, we explore the possibility of absorbing the fourth-order correction,

$$
E_{4}=E_{\hbar^{4}}^{C N}(n)+E_{\hbar^{4}}^{S O}(n)+E_{\hbar^{4}}^{C N}(p)+E_{\hbar^{4}}^{S O}(p)
$$

into the net second-order contribution,

$$
E_{2}=E_{\hbar^{2}}^{C N}(n)+E_{\hbar^{2}}^{S O}(n)+E_{\hbar^{2}}^{C N}(p)+E_{\hbar^{2}}^{S O}(p) .
$$

Here, $(n)$ and $(p)$ stand for neutronic and protonic contributions. See Eqs. (11)-(14) for the definitions of the different terms appearing in these two equations. Clearly, if such an absorption is possible, the factor $\alpha$ [see Eqs. (1) and (2) for definition], should be expressible as a function of neutron number, proton number, or some combinations thereof. Before discussing the possibility of absorbing fourth-order terms into second-order terms for a Woods-Saxon potential, we demonstrate the existence of such a functional form for the simple harmonic oscillator potential.

\section{A. The harmonic oscillator potential}

The harmonic oscillator $(\mathrm{HO})$ potential provides a unique opportunity to investigate the details of the WK expansions analytically. Therefore, first we consider the simplest form of the HO potential, without spin-orbit interaction. It can be shown that, for the HO potential, the different WK corrections are given by Ref. [10]

$$
\begin{aligned}
& E_{4}=\frac{-17 \hbar \omega}{960}, \\
& E_{2}=\frac{\lambda^{2}}{8 \hbar \omega},
\end{aligned}
$$

where $\lambda$ is the chemical potential, determined as described earlier, and $\omega$ is the oscillator frequency. For the HO potential, assuming degeneracy of 2, the particle number [see Eq. (7)] is given by

$$
\mathcal{N}=\frac{1}{3}\left(\frac{\lambda}{\hbar \omega}\right)^{3}-\frac{1}{4}\left(\frac{\lambda}{\hbar \omega}\right) .
$$

This equation is cubic in $\lambda /(\hbar \omega)$, and in principle can be solved exactly. Here, however, we take an alternative and physically more transparent approach, wherein, we express $\lambda$ as $[15,43]$

$$
\lambda=\lambda_{0}+\lambda_{2}+\lambda_{4},
$$

where $\lambda_{j}$ is correct up to order $\hbar^{j}$. Starting from the ThomasFermi expression for the chemical potential, and noticing that the normalization is true order by order, we get the following 
expression for chemical potential, correct up to $\hbar^{4}$ :

$$
\lambda=\left\{(3 \mathcal{N})^{1 / 3}+\frac{1}{4}(3 \mathcal{N})^{-1 / 3}\right\} \hbar \omega .
$$

This, along with the second and fourth-order WK corrections to energy [see Eqs. (11) and (12)], yields

$$
\alpha=1-\frac{17}{60} \frac{(\hbar \omega)^{2}}{\lambda_{p}^{2}+\lambda_{n}^{2}}
$$

where $\lambda_{p}$ and $\lambda_{n}$ are chemical potentials for $Z$ protons and $N$ neutrons respectively. Further, notice that the neutron and proton numbers can be written as

$$
N=\frac{1+I}{2} A \quad \text { and } \quad Z=\frac{1-I}{2} A
$$

$A=N+Z$ being the mass number of the nucleus, and $I$ being the asymmetry parameter, defined as $I=(N-Z) / A$. We obtain

$$
\alpha=1-\frac{17}{120}\left(\frac{2}{3}\right)^{2 / 3} A^{-2 / 3}\left(1+\frac{1}{9} I^{2}\right),
$$

where the terms up to the order $A^{-2 / 3}$ are retained, and the expansion in $I$ has been carried out only up to second order in $I$. It can be therefore seen that the factor $\alpha$ can indeed be written as a function of mass number and $I$, implying that it is in principle possible, at least in the case of the HO potential, to absorb the fourth-order WK corrections to the energy into the second-order WK corrections.

To understand the behavior of $\alpha$ with respect to $I$, we plot the factor $\alpha$ as a function of $I$ in Fig. 8. It is seen that the factor $\alpha$ has a very regular behavior with respect to asymmetry. There are points stacked at a given value of $I$, with groups of points placed symmetrically with respect to them. This regularity persists over the entire range of $I$ values.

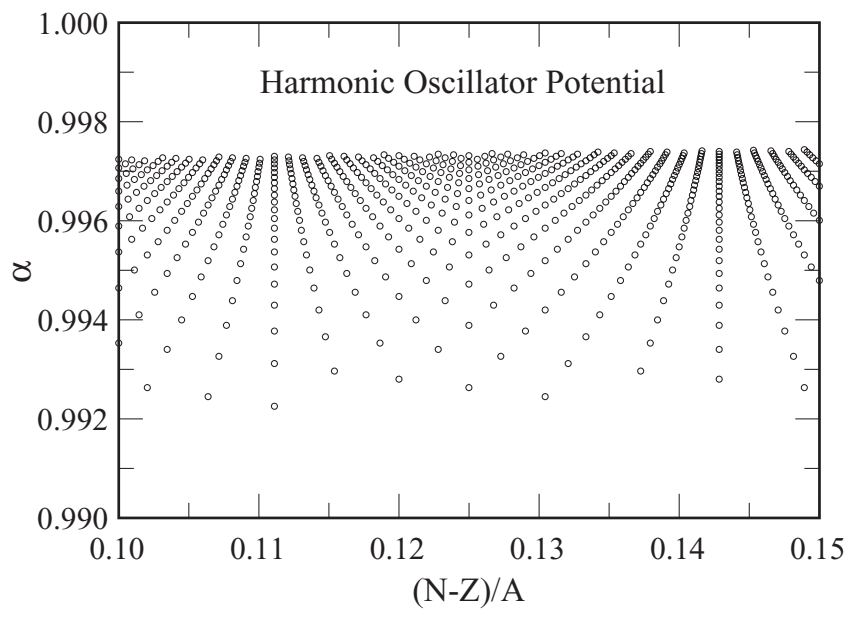

FIG. 8. The factor $\alpha$ for a harmonic oscillator potential, without spin-orbit interaction. Only a small portion of the asymmetry scale has been presented.

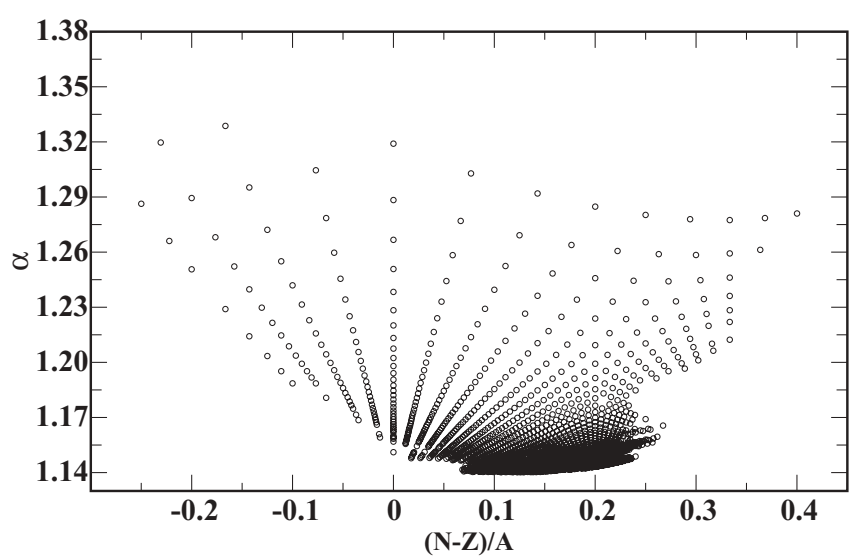

FIG. 9. Factor $\alpha$ as a function of asymmetry for a Woods-Saxon potential.

\section{B. Woods-Saxon potential}

Next, we investigate the factor $\alpha$ for the Woods-Saxon potential. In order to achieve this, we choose a set of 2171 known nuclei [25] with $Z>5$. Spherical symmetry is assumed. The nuclear, spin-orbit, and Coulomb potentials have been taken as defined in Ref. [6]. The full Wigner-Kirkwood calculations up to the fourth order in $\hbar$ are carried out for these nuclei, and the exact values of the factor $\alpha$ are obtained. These are then plotted as a function of the asymmetry parameter $I$ in Fig. 9. The figure exhibits that the factor $\alpha$ has a very regular behavior as a function of asymmetry. In order to understand the detailed structure of the factor $\alpha$, we plot the same results with a greater resolution in Fig. 10.

A remarkable and regular pattern emerges from the plots. In comparison with the case of the HO potential, the pattern is inverted. The pattern consists of "fanlike" structures. There are groups of points stacked exactly along vertical lines, as indicated in Fig. 10, accompanied by symmetrically placed, slanting groups of points. All these groups of points constitute nearly perfect straight lines. This is in contrast with the case of the $\mathrm{HO}$ potential, where the lines were curved.

A closer examination of the behavior of the factor $\alpha$ reveals several interesting features. To understand them better, we shall first enlist the nuclei appearing in a particular "fan" structure. We shall designate the slanting lines appearing in the fan structure as "rays." Thus, each fan structure has a number of rays in it, symmetrically placed with respect to the vertical line, defined by a particular ratio, $(N-Z) / A$. For example, let us consider $(N-Z) / A=1 / 11$. This fan structure has ${ }^{22} \mathrm{Ne}$, ${ }^{33} \mathrm{P},{ }^{44} \mathrm{Ca}, \ldots,{ }^{176} \mathrm{Hg}, \ldots$ along the vertical line. The first ray to the right of this line contains nuclei such as ${ }^{20} \mathrm{~F},{ }^{31} \mathrm{Si},{ }^{42} \mathrm{~K}, \ldots$. The second ray to the right of the vertical line consists of the nuclei such as ${ }^{40} \mathrm{Ar},{ }^{51} \mathrm{~V},{ }^{62} \mathrm{Ni}$ etc. The first ray to the left of the vertical line consists of ${ }^{35} \mathrm{~S},{ }^{46} \mathrm{Sc},{ }^{57} \mathrm{Fe}$, etc., whereas, the second ray to the left of the vertical line consists of ${ }^{37} \mathrm{Cl},{ }^{48} \mathrm{Ti}$, ${ }^{59} \mathrm{Co}$, etc. The heavier nuclei in this sequence are towards the bottom of the pattern. The value of $\alpha$ is therefore, inversely proportional to the mass number. Thus, it is expected that in the limit of $A \rightarrow \infty$, the $\alpha$ values will approach some constant value, say, $\alpha_{0}$, which is approximately 1.125 , according to the figure above. 

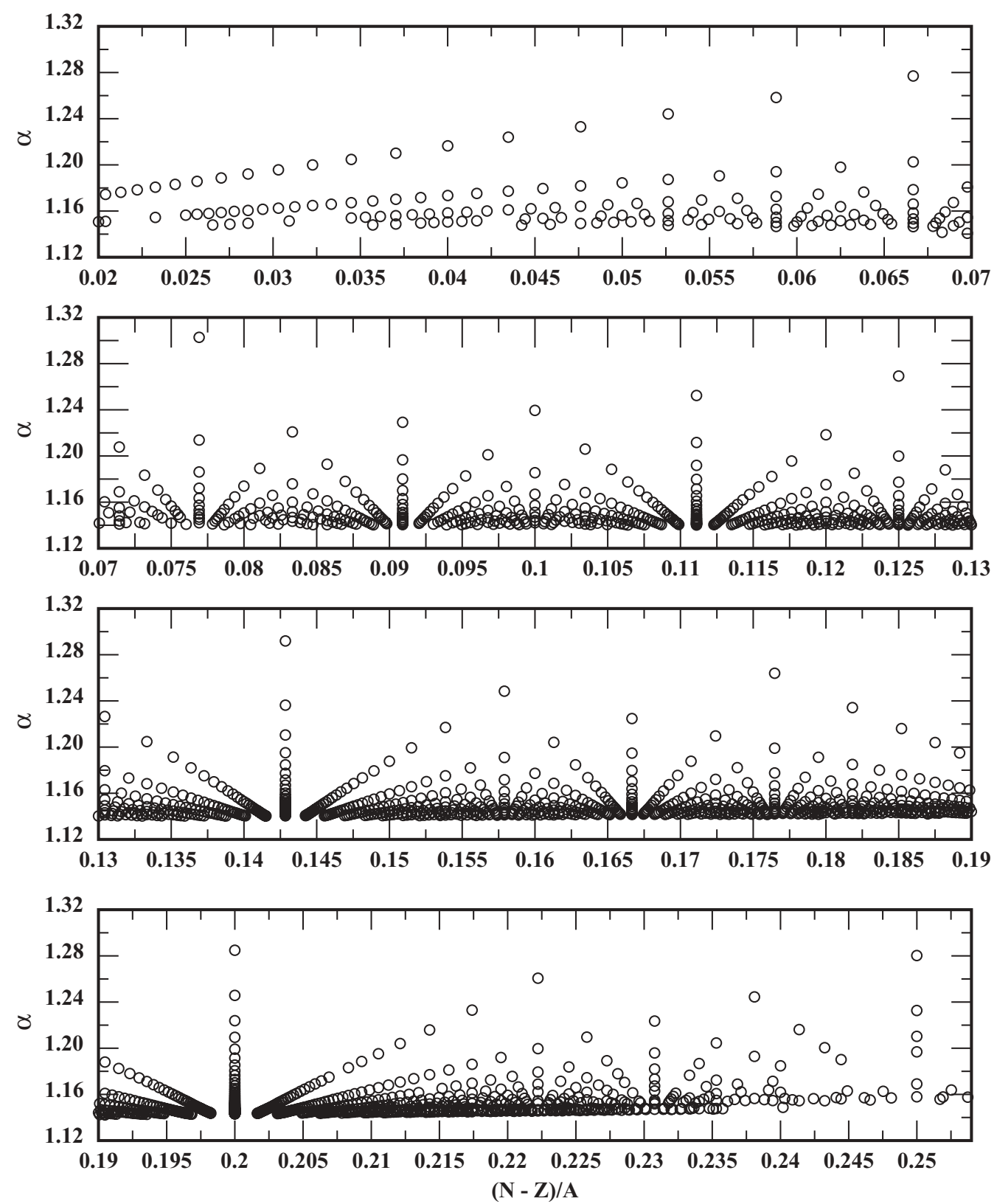

FIG. 10. Fate $\alpha$ as a function of asymmetry.

Considering these observations, we propose the following parametrization for the factor $\alpha$ :

$$
\alpha=\alpha_{0}+\frac{\alpha_{1}}{A}+\alpha_{2} \frac{N-Z}{A}+\alpha_{3}\left(\frac{N-Z}{A}\right)^{2},
$$

where $\alpha_{j}$ 's are adjustable parameters. Considering all the 2171 nuclei (see above), we carry out a least-squares fit to determine these parameters. The fit turns out to be exceptionally good, with rms deviation $1.09 \times 10^{-3}$. The values of the parameters are $\alpha_{0}=1.12761, \alpha_{1}=2.26744$, $\alpha_{2}=-0.02659$, and $\alpha_{3}=0.29987$. The difference between the exact and the corresponding fitted $\alpha$ values is plotted in Fig. 11, indicating that the agreement is almost perfect, and that the phenomenological formula that has been proposed here is indeed robust, for all the mass regions.

We shall now investigate the deformation effects, particularly with reference to the factor $\alpha$. In order to achieve that, we once again consider the set of 561 even-even nuclei (see Sec. III), with deformation parameters obtained as described before. The calculation of binding energies requires the shell corrections, pairing energies, and the liquid-drop energies. The shell corrections require averaged energies, which are calculated here using the WK expansion. Here, we consider the WK expansion only up to second order, and simulate the effects of fourth order through the factor $\alpha$ [Eq. (34)]. This defines the averaged energies and hence the shell corrections completely. The difference between the shell corrections thus obtained and 


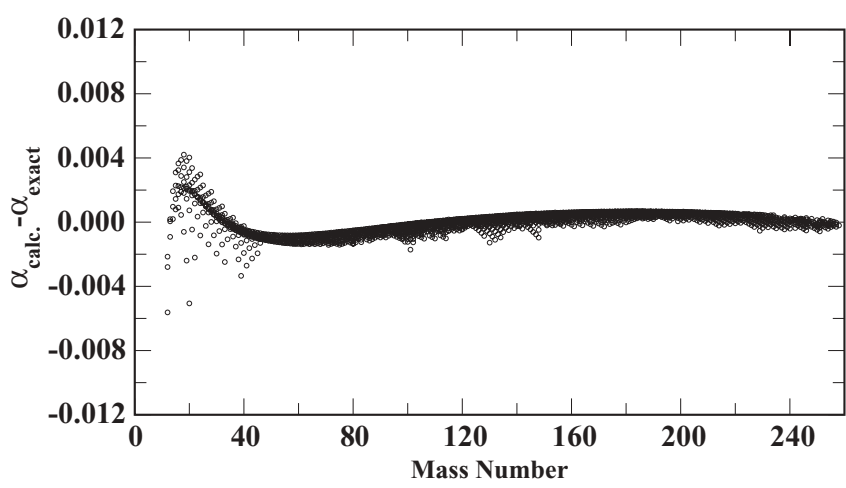

FIG. 11. The difference between the fitted and the corresponding exact values of $\alpha$.

the "exact" shell corrections is found to be indeed small, the maximum deviation being of the order $150 \mathrm{keV}$, implying that the factor $\alpha$ obtained merely by using the spherical nuclei works very well for deformed systems as well (with both deformation parameters $\beta_{2}$ and $\beta_{4}$ ). This observation is indeed of great practical importance.

With these approximate shell corrections, we make a refit to the liquid-drop parameters. Comparison between the liquid-drop parameters as reported in Sec. III and the ones obtained with the approximate shell corrections is presented in Table III. It is indeed gratifying to note that the liquid-drop parameters obtained in the two cases are almost identical, and so is the rms deviation of the calculated binding energies with respect to experiment [25]. This substantiates the validity of the parametrization of $\alpha$.

To test the robustness of the parametrization of $\alpha$ further, we calculate the constants $\alpha_{j}$ 's in Eq. (34) using just four nuclei $\left({ }^{40} \mathrm{Ca},{ }^{100} \mathrm{Sn},{ }^{146} \mathrm{Gd}\right.$, and $\left.{ }^{208} \mathrm{~Pb}\right)$ instead of 2171 nuclei as described above. It is found that the numerical values of the constants practically remain the same. To test the validity of these parameters, the liquid-drop parameters are reworked employing the new values of $\alpha_{j}$ 's. It is found that the liquiddrop parameters thus obtained are practically equal to the ones reported in the right-most column of Table III.

We close this section by concluding that the absorption of fourth-order Wigner-Kirkwood corrections into the second contributions is reliable, and can be used in large scale

TABLE III. Values of the liquid drop parameters obtained through the $\chi^{2}$ minimization for exact and approximate shell corrections.

\begin{tabular}{lrr}
\hline \hline Quantity & \multicolumn{1}{c}{ Exact } & Approx. \\
\hline$a_{v}$ & -15.435 & -15.421 \\
$k_{v}$ & -1.875 & -1.873 \\
$a_{s}$ & 16.673 & 16.580 \\
$k_{S}$ & -2.430 & -2.432 \\
$a_{\text {cur }}$ & 3.161 & 3.295 \\
$r_{0}$ & 1.219 & 1.221 \\
$C_{4}$ & 0.963 & 0.953 \\
$w_{1}$ & -2.763 & -2.652 \\
$w_{2}$ & 3.725 & 3.659 \\
$\mathrm{rms}$ & 0.610 & 0.607 \\
\hline \hline
\end{tabular}

mic-mac calculations. The absorption also has the advantage of reducing the numerical noise that might arise in the higher-order derivatives of the potentials.

\section{SUMMARY AND CONCLUSIONS}

The semiclassical Wigner-Kirkwood $\hbar$ expansion of the one-body partition function has been employed instead of the Strutinsky averaging scheme to calculate the shell corrections within the framework of a mic-mac model. The microscopic part of the energy also contains pairing contributions that are obtained using the Lipkin-Nogami scheme. We have improved the macroscopic part of the model as compared with the one used in our previous work [6,7] by including the curvature term as well as the Wigner contribution. With just ten adjustable parameters, our model reproduces the binding energies of 561 even-even spherical and deformed nuclei with rms deviation of $610 \mathrm{keV}$. We have tested this new mic-mac model near the proton and neutron drip lines as well as in the superheavy region. Our present calculations show that the mic-mac model proposed in this paper reproduces remarkably well the recent experimental results in these exotic scenarios.

Further, a systematic study of the ratio of the fourth-order and second-order Wigner-Kirkwood energies has been carried out. We find that the ratio of these two energies behaves in a very systematic manner. We have shown that this ratio can be parametrized accurately by a simple expression, implying that the fourth-order corrections can be absorbed into the secondorder contributions in a very simple way. We have checked that, using this simple procedure, we recover practically the same parameters of the macroscopic part, without deterioration of the quality of agreement achieved with the full WignerKirkwood calculation including explicitly the fourth-order contributions. Therefore, this simplified calculation of shell corrections can be used confidently in the large-scale mic-mac calculations that we plan to carry out as the next step.

Finally, we point out that there is still some room for improving our model, particularly in two specific directions. On the one hand, the full blocking procedure in the pairing calculations of odd-odd, odd-even, and even-odd nuclei, which may be particularly relevant for spherical nuclei, has to be introduced. On the other hand, refinements in the meanfield Woods-Saxon potential and in the distance function are still needed to study with our model not only neutron-rich nuclei, but also fission barriers. This would require large-scale calculations with the model, for which the simplification proposed above may be very useful.

\section{ACKNOWLEDGMENTS}

A.B. acknowledges partial financial support from Department of Science and Technology, Government of India (Grant No. SR/S2/HEP-34/2009). M.C. and X.V. were partially supported by the Consolider Ingenio 2010 Programme CPAN CSD2007-00042, Grant No. FIS2011-24154 from MICINN and FEDER, and Grant No. 2009SGR-1289 from Generalitat de Catalunya. 
[1] M. Bender, P.-H. Heenen, and P.-G. Reinhard, Rev. Mod. Phys. 75, 121 (2003).

[2] D. Lunney, J. M. Pearson, and C. Thibault, Rev. Mod. Phys. 75, 1021 (2003).

[3] J. R. Stone and P.-G. Reinhard, Prog. Part. Nucl. Phys. 58, 587 (2007).

[4] W. D. Myers, Droplet Model of Atomic Nuclei (Plenum, New York, 1977); W. D. Myers and W. J. Swiatecki, Ann. Phys. (NY) 55, 395 (1969); 84, 186 (1974).

[5] K. Pomorski and J. Dudek, Phys. Rev. C 67, 044316 (2003).

[6] A. Bhagwat, X. Viñas, M. Centelles, P. Schuck, and R. Wyss, Phys. Rev. C 81, 044321 (2010).

[7] A. Bhagwat, R. Wyss, X. Viñas, and P. Schuck, Int. J. Mod. Phys. E 19, 747 (2010).

[8] E. Wigner, Phys. Rev. 40, 749 (1932).

[9] J. G. Kirkwood, Phys. Rev. 44, 31 (1933).

[10] B. K. Jennings, R. K. Bhaduri, and M. Brack, Nucl. Phys. A 253, 29 (1975).

[11] P. Ring and P. Schuck, The Nuclear Many-Body Problem (Springer-Verlag, Berlin, 1980).

[12] M. Brack, C. Guet, and H.-B. Håkansson, Phys. Rep. 123, 275 (1985).

[13] M. Brack and R. K. Bhaduri, Semi-classical Physics (AddisonWesley, Reading, MA, 1997).

[14] M. Centelles, P. Leboeuf, A. G. Monastra, J. Roccia, P. Schuck, and X. Vinas, Phys. Rev. C 74, 034332 (2006), and references cited therein.

[15] M. Centelles, P. Schuck, and X. Viñas, Ann. Phys. (NY) 322, 363 (2007).

[16] B. Mohammed-Azizi and D. E. Medjadi, Phys. Rev. C 74, 054302 (2006).

[17] P. Schuck and X. Viñas, Phys. Lett. B 302, 1 (1993).

[18] X. L. Tu et al., Phys. Rev. Lett. 106, 112501 (2011).

[19] Y. Oganessian, J. Phys. G: Nucl. Part. Phys. 34, R156 (2007).

[20] H. J. Lipkin, Ann. Phys. (NY) 9, 272 (1960).

[21] Y. Nogami, Phys. Rev. 134, B313 (1964).

[22] H. C. Pradhan, Y. Nogami, and J. Law, Nucl. Phys. A 201, 357 (1973).
[23] P. Möller, J. R. Nix, W. D. Myers, and W. J. Swiatecki, At. Data Nucl. Data Tables 59, 185 (1995).

[24] R. S. Kurmanov, G. I. Kosenko, and G. D. Adeev, Yad. Fiz. 63, 1978 (2000) [Phys. At. Nucl. 63, 1885 (2000)].

[25] G. Audi, A. H. Wapstra, and C. Thibault, Nucl. Phys. A 729, 337 (2003).

[26] See Supplemental Material at http://link.aps.org/supplemental/ 10.1103/PhysRevC.86.044316 for the table.

[27] P. Möller, J. R. Nix, and K.-L. Kratz, At. Data Nucl. Data Tables 66, 131 (1997).

[28] I. Angeli, At. Data Nucl. Data Tables 87, 185 (2004).

[29] M. Hemalatha, A. Bhagwat, A. Shrivastava, S. Kailas, and Y. K. Gambhir, Phys. Rev. C 70, 044320 (2004).

[30] J. P. Maharana, Y. K. Gambhir, J. A. Sheikh, and P. Ring, Phys. Rev. C 46, R1163 (1992), and references cited therein.

[31] D. C. Zheng and L. Zamick, Phys. Lett. B 266, 5 (1991).

[32] R. Sahu and S. P. Pandya, J. Phys. G 16, 429 (1990), and references therein.

[33] P. Haensel, A. Y. Potekhin, and D. G. Yakovlev, Neutron Stars 1: Equation of State and Structure (Springer, New York, 2007).

[34] S. B. Rüster, M. Hempel, and J. Schaffner-Bielich, Phys. Rev. C 73, 035804 (2006).

[35] X. Roca-Maza and J. Piekarewicz, Phys. Rev. C 78, 025807 (2008).

[36] X. Roca-Maza, J. Piekarewicz, T. García-Gálvez, and M. Centelles, arXiv:1109.3011.

[37] M. Bender, Phys. Rev. C 61, 031302 (2000).

[38] S. Goriely, M. Samyn, P. H. Heenen, J. M. Pearson, and F. Tondeur, Phys. Rev. C 66, 024326 (2002).

[39] I. Muntian, Z. Patyk, and A. Sobiczewski, Phys. At. Nucl. 66, 1015 (2003)

[40] Y. K. Gambhir, A. Bhagwat, and M. Gupta, Ann. Phys. (NY) 320, 429 (2005).

[41] S. Hoffman and G. Munzenburg, Rev. Mod. Phys. 72, 733 (2000).

[42] V. E. Viola Jr. and G. T. Seaborg, J. Inorg. Nucl. Chem. 28, 741 (1966).

[43] M. Centelles, X. Viñas, M. Durand, P. Schuck, and D. Von-Eiff, Ann. Phys. (NY) 266, 207 (1998). 